Scientific Paper

Doi: http://dx.doi.org/10.1590/1809-4430-Eng.Agric.v42n1e20210129/2022

\title{
DESIGN AND EXPERIMENT OF A SAFFLOWER PICKING ROBOT BASED ON A PARALLEL MANIPULATOR
}

\author{
Hui Guo ${ }^{1 *}$, Dan Luo ${ }^{1}$, Guomin Gao ${ }^{1}$, Tianlun $\mathrm{Wu}^{1}$, Hongwei Diao ${ }^{1}$ \\ ${ }^{1 *}$ Corresponding author. College of Mechanical and Electrical Engineering, Xinjiang Agricultural University/ Urumqi, China. \\ E-mail: gh97026@163.com | ORCID ID: http://orcid. org /0000-0001-6877-9995
}

\section{KEYWORDS}

Picking robot, Parallel manipulator, Safflower, Kinematics analysis, Net picking rate.

\begin{abstract}
To mitigate the large demand for safflower picking labour and the low efficiency of manual picking, a safflower picking robot that is based on a parallel manipulator is designed. The whole robot mainly consists of a walking device, parallel manipulator device, vision device, picking device, filament collection device, control system, and motor drive system. The forward and inverse kinematics analysis of the parallel manipulator is analysed via a geometric method, the kinematics model is established, and the computational analysis of the parallel manipulator working speed is based on the Jacobi matrix. For driving angle errors of $0.001 \sim 0.005^{\circ}$, the moving platform's motion accuracy is $0.2174-0.9387 \mathrm{~mm}$, which meets the position accuracy requirements of the test stage. Based on MATLAB software, the Monte Carlo method is employed to solve the working space of the parallel manipulator, which is approximately an equilateral triangle with a side length of $0.35 \mathrm{~m}$, which satisfies the safflower growth space picking conditions. The prototype is constructed, and safflower picking experiments are carried out under laboratory conditions. The average picking period of each flower bulb was $16 \mathrm{~s}$, and the average net picking rate of the filaments was $87.91 \%$. The experimental results verified the applicability of the safflower picking robot.
\end{abstract}

\section{INTRODUCTION}

Safflower, also known as a red and blue flower, is a special economic crop that integrates medicinal and oil plants and exhibits the promotion of blood circulation, clearing of blood stasis and relieving of pain (State Pharmacopoeia Committee, 2010). According to the growth characteristics of safflower plants, each flower bulb had three batches of flowering time, and the flowering time of each batch was 4 to 5 days. Therefore, the entire safflower silk picking cycle generally lasts from 12 to 16 days ( $\mathrm{Li} \& \mathrm{Li}, 2011$ ). Presently, the safflower picking operation needs to be completed manually. Repeated harvesting has increased the working time and labour intensity of workers. The safflower harvesting time for the summer overlaps with the harvesting time of other cash crops. The labour supply exceeds the demand, and it is difficult to achieve an economic time-efficient harvesting effect. The problem of safflower harvesting is already imminent, and the realization of automation and mechanization of safflower picking is a necessary condition for vigorously developing the safflower industry. Presently, research on safflower picking machinery at home and abroad is at the preliminary stage. In recent years, relevant technical research has been carried out. The existing safflower harvesting machinery mainly adopts rollers (Zhang et al., 2020b), knife cutting (Bai et al., 2020; Liu et al., 2019) or combs (Sun et al., 2018; An et al., 2020; Lian et al., 2019) to complete the separation of filaments and fruit balls (Siavash et al., 2012). India Anil (2005) successively proposed two pneumatic harvesters (Mc Guire et al., 2012). A battery is used to power the fan, and the fan generates negative pressure to suck the filaments into the pipe. With the wind, the filaments are drawn into the filament collection room, which improves the picking efficiency, but the structure is bulky and needs to be carried manually. Cao et al. (2018) examined a comb-tooth safflower picking device, which makes the

${ }^{1}$ College of Mechanical and Electrical Engineering, Xinjiang Agricultural University/ Urumqi, China. 
flower ball relatively fixed by the timing action of the limiting bar and comb teeth. The filaments are clamped and rotated by the comb teeth to realize self-propelled picking, which has the characteristics of reducing labour intensity and improving harvesting efficiency, but the impurity content of filaments is relatively high. Zhang et al. (2019) designed a rotary shear-type safflower picker, which cut the filaments by rotating the picking manipulator and subsequently collected the filaments under the negative pressure generated by the negative pressure fan. Although the filament breakage rate is reduced, the picking efficiency can be improved. The abovementioned research shows that corresponding machines for safflower picking exist, but substantial manual assistance is still needed. To realize automatic and intelligent picking, this paper designs a safflower picking robot based on a parallel manipulator to realize the identification, positioning and picking collection of safflowers to improve the efficiency, accuracy and automation of safflower picking.

\section{Structure and working principle}

\section{Main structure}

The row width of standardized planted safflower plants is $450 \mathrm{~mm}$; the row spacing is $50 \mathrm{~mm}$; the average height of safflower plants is $777.91 \mathrm{~mm}$; and the maximum horizontal width of the plant in the natural state is 274.5 $\mathrm{mm}$ (Han, 2014). The width and height of the safflower picking machine designed in this paper are $1.2 \mathrm{~m}$ and 1.3 $\mathrm{m}$, respectively. As shown in Fig. 1, the machine is composed of a walking device, parallel manipulator device, vision device, picking device, filament collection device, control system and motor drive system.

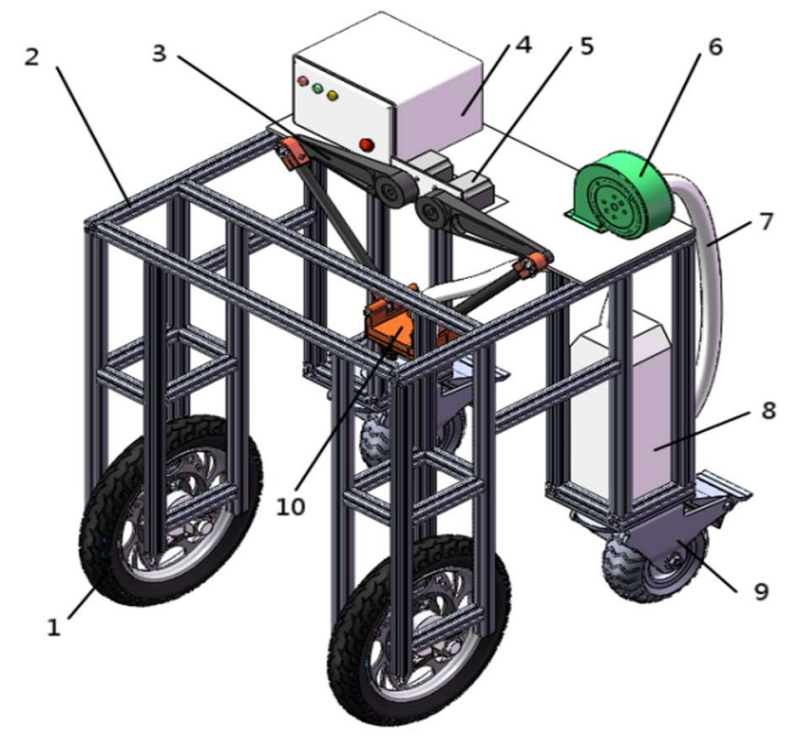

(1) electric drive wheels, (2) frame,

(3) parallel manipulator device, (4) controller, (5) servo motor, (6) negative pressure suction fan, (7) house,

(8) filament collection box, and (9) walking wheels.

FIGURE 1. Structure diagram of the safflower picking machine.

\section{Working principle}

The working principle of the whole machine is described as follows. The power is turned on during operation, and the walking device controls the forward, backward, stop, and steering of the whole machine. The whole machine travels among the rows of safflower plants and pauses when it reaches the mature safflower area to be harvested. The upper (host) computer controls the motor rotation, where the motor shaft is connected to the active arm in the parallel manipulator device, and the motor shaft rotates to a certain angle to drive the parallel manipulator to move to the target position. The safflower enters the picking device with the flower conveying hole, and the crank slider shearing device performs reciprocating shearing of the safflower. The filament collecting device is powered, the suction fan generates negative pressure, and the filament is sucked into the collection box and passes through the flower conveying hose under negative pressure, completing the picking and collection of safflower filaments.

\section{Design of the parallel manipulator}

A parallel mechanism refers to a closed, multiloop mechanism with multiple degrees of freedom that is distributed on different loops (Huang et al., 1997). Compared with tandem robots, the parallel mechanism has the characteristics of adequate stiffness, high accuracy, high speed, etc. Therefore, over time, the parallel mechanism has become a research hotspot in the field of robotics. The two-degree-of-freedom (2-DOF) parallel manipulator is one of the important devices of the whole machine. Each branch chain of the parallel mechanism is relatively independent and does not affect each other. The end of each branch chain is simultaneously connected and fixed with the moving platform, where the moving platform is equivalent to the end output of the tandem robot (Ma et al., 2006). Working principle: The motor starts to work independently after receiving the control signal from the host computer; the motor shaft drives the two active arms in the parallel mechanism to rotate the specified angle; the active arm drives the slave arm to work, and the two parallel branch chains concurrently drive the end picking device and move until the picking device reaches the target position. The safflower bulbs then enter the picking device through the flower conveying hole and are fixed. The structure of the parallel manipulator device is shown in Fig. 2.

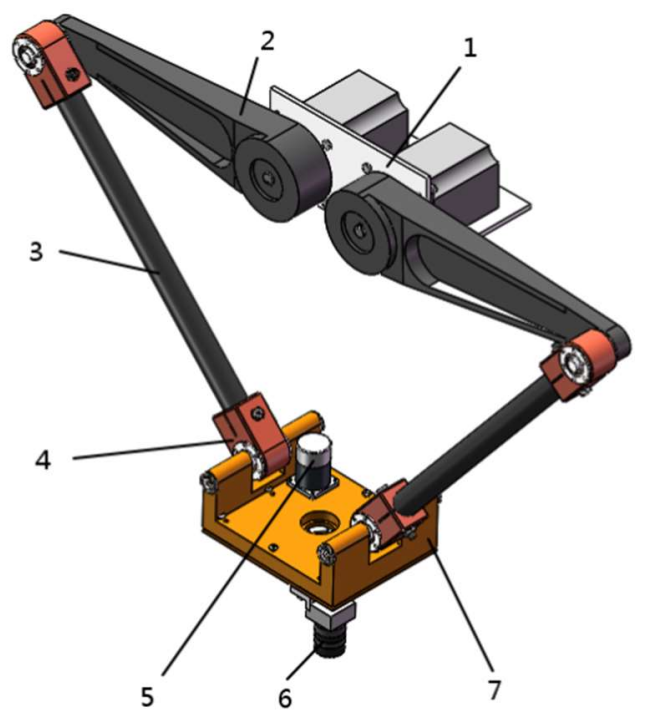

(1) motor base, (2) active arm, (3) slave arm, (4) adapter, (5) stepping motor, (6) CCD camera, and (7) picking device.

FIGURE 2. Parallel manipulator structure diagram. 


\section{Kinematics analysis of the Parallel mechanism}

\section{Inverse kinematics analysis}

During the picking process, the position coordinates of the safflower are judged in advance, and the stepping motor is subsequently controlled to drive the parallel mechanical arm device to the target position through the rotation of the two motor shafts. An inverse kinematics analysis is performed to calculate the joint variables of each joint by knowing the pose of the end effector of the robotic arm (Wang et al., 2020). Many methods are available to solve inverse kinematics; these methods can be divided into analytical methods and numerical methods, of which the analytical methods include algebraic methods and geometric methods (Zhang et al., 2020a; Zhao et al., 2020). The experiment employed a geometric method to establish the plane coordinate system $O-X Y$ (Kuo et al., 2021). The parallel mechanical arm is simplified as a plane linkage mechanism. The length of each linkage in the plane linkage mechanism is $l_{1}, l_{2}, l_{3}, l_{4}, l_{5}, l_{6}$, and $l_{7}$, where rods $l_{1}$ and $l_{6}$ are stepping motor drive rods, that is, the active arms of the two parallel branch chains, as shown in Fig. 3.

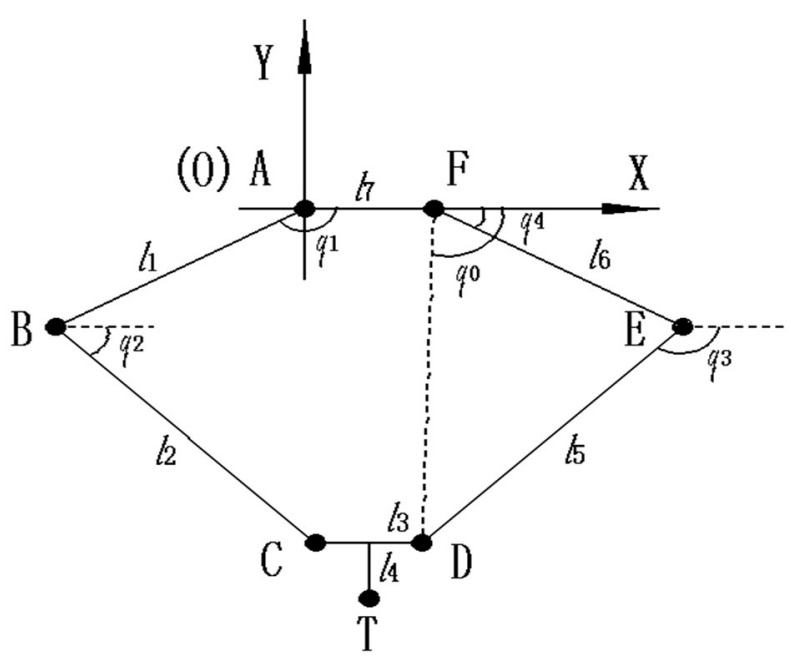

FIGURE 3. Inverse kinematic analysis of the planar mechanism of the parallel manipulator.

The two points $\mathrm{A}$ and $\mathrm{F}$ are fixed, and point $\mathrm{A}$ coincides with origin $\mathrm{O}$ of the Cartesian coordinate system. It is assumed that point $\mathrm{T}$ is the centre point of the shear hole position of the end picking device, so the position coordinates of point $\mathrm{T}$ are known, and the position angle $q_{1}$ of the two parallel branch chains and $q_{4}$ are solved in reverse.

The analysis diagram shows the position of the $T$ point and the position coordinate relationship of the $\mathrm{D}$ point:

$$
\begin{gathered}
D_{X}=T_{X}+l_{3} \\
D_{Y}=T_{Y}+l_{4}
\end{gathered}
$$

Solve for the distance of the FD connection:

$$
\rho_{D}=\sqrt{\left(D_{X}-F_{X}\right)^{2}+\left(D_{Y}-F_{Y}\right)^{2}}
$$

$$
q_{0}=\arctan \left(\frac{D_{Y}-F_{Y}}{D_{X}-F_{X}}\right)
$$

When:

$\rho_{\mathrm{D}}$ and $q_{0}$ are obtained, the coordinates of points $\mathrm{E}$ and $\mathrm{D}$ can be solved:

$$
\begin{aligned}
& E_{X}=l_{7}+l_{6} \cos \left[q_{0} \pm \cos ^{-1}\left(\frac{\rho_{D}^{2}+l_{6}^{2}-l_{5}^{2}}{2 \rho_{D} l_{6}}\right)\right] \\
& E_{Y}=l_{6} \sin \left[q_{0} \pm \cos ^{-1}\left(\frac{\rho_{D}^{2}+l_{6}^{2}-l_{5}^{2}}{2 \rho_{D} l_{6}}\right)\right] \\
& D_{X}=E_{X}+l_{5} \cos q_{3} \\
& D_{Y}=E_{Y}+l_{5} \sin q_{3}
\end{aligned}
$$

Introduce eqs (3), (4), (5) and (6) to solve for $q_{3}$ :

$$
q_{3}=\tan ^{-1}\left(\frac{\rho_{D} \sin q_{0}-E_{Y}}{\rho_{D} \cos q_{0}-E_{X}+l_{7}}\right)
$$

in which:

$\mathrm{T}_{\mathrm{X}}$ - transverse coordinate of the centre point of the shear hole, mm;

$T_{Y}$ - transverse coordinate of the centre point of the shear hole, mm;

$\rho_{D}$-length of FD line segment, mm;

$\mathrm{D}_{\mathrm{X}}$ - transverse coordinate of point $\mathrm{D}, \mathrm{mm}$;

$\mathrm{C}_{\mathrm{Y}}$ - longitudinal coordinate of point $\mathrm{D}, \mathrm{mm}$;

$\mathrm{F}_{\mathrm{X}}$ - transverse coordinate of point $\mathrm{F}, \mathrm{mm}$;

$\mathrm{F}_{\mathrm{Y}}$ - longitudinal coordinate of point $\mathrm{F}, \mathrm{mm}$;

$q_{0}$ - angle between the FD line segment and the Xaxis, $\left(^{\circ}\right)$;

$l_{3}$ - radius of the end picking device, $\mathrm{mm}$;

$l_{4}$ - distance between the end platform and the centre of the shear hole, $\mathrm{mm}$,

$l_{5}, l_{6}, l_{7}$ - values of rod ED, FE, and AF, mm.

Equations (1) and (2) are input into eqs (3) and (4), respectively, to obtain the length of the FD line segment and $q_{0}$ and then input into eqs (5) and (6), respectively, to obtain the coordinates of point $\mathrm{E}$ and further solve for $q_{1}$ and $q_{4}$.

$$
\begin{gathered}
C_{X}=T_{X}-l_{3} \\
C_{Y}=T_{Y}+l_{4}
\end{gathered}
$$

$$
q_{1}=\tan ^{-1}\left(\frac{C_{Y}}{C_{X}}\right) \pm \cos ^{-1}\left(\frac{C_{X}^{2}+C_{Y}^{2}+l_{1}^{2}-l_{2}^{2}}{2 \sqrt{C_{X}^{2}+C_{Y}^{2}} \cdot l_{1}}\right)
$$




$$
q_{4}=q_{0} \pm \cos ^{-1}\left(\frac{\rho_{D}^{2}+l_{6}^{2}-l_{5}^{2}}{2 \rho_{D} l_{6}}\right)
$$

in which:

$\mathrm{C}_{\mathrm{X}}$ - horizontal coordinate of point $\mathrm{C}, \mathrm{mm}$;

$\mathrm{C}_{\mathrm{Y}}$ - longitudinal coordinate of point $\mathrm{C}, \mathrm{mm}$;

$l_{1}, l_{2}-\operatorname{rod}$ lengths of rods $\mathrm{AB}$ and $\mathrm{BC}, \mathrm{mm}$;

$q_{1}$ - angle of rotation of active $\operatorname{rod} \mathrm{AB},\left(^{\circ}\right)$,

$q_{4}$-angle of rotation of active $\operatorname{rod} \mathrm{FE},\left(^{\circ}\right)$.

\section{Speed analysis}

In the control of the parallel manipulator mechanism, the speed of the parallel manipulator needs to be analysed so that the speed of the central reference point of the end picking device can be obtained. On this basis, the speed Jacobi matrix of the parallel manipulator is obtained, and the speed Jacobi matrix is the linear transformation between the generalized output speed and the generalized input speed of the robot mechanism, usually denoted as $J_{T}(q)$ (Dai et al., 2016; Hoevenaars et al., 2020).

$J_{T}(q)$ is the Jacobi matrix of the end picking device and the active arm in the parallel manipulator, the derivative method is utilized to obtain the Jacobi matrix, the equation of the relationship between the input position and the output position has been established; and then the derivative for time is obtained $J_{T}(q)$. The end picking device shear hole centre point $T$ is selected for analysis and calculation, and $q$ is established as two active input parameters $\left(\dot{q_{1}}, \dot{q_{4}}\right)^{T}$.

For parallel branch chain $\mathrm{ABC}$ analysis:

$$
\begin{aligned}
& \left\{\begin{array}{l}
\dot{C}_{X}=-l_{1} \sin q_{1} \dot{q}_{1}-l_{2} \sin q_{2} \dot{q}_{2} \\
\dot{C}_{Y}=-l_{1} \cos q_{1} \dot{q}_{1}+l_{2} \cos q_{2} \dot{q}_{2}
\end{array}\right. \\
& \left\{\begin{array}{l}
\dot{D}_{X}=-l_{6} \sin q_{4} \dot{q}_{4}-l_{6} \sin q_{3} \dot{q}_{3} \\
\dot{D}_{Y}=l_{6} \cos q_{4} \dot{q}_{4}+l_{6} \cos q_{3} \dot{q}_{3}
\end{array}\right.
\end{aligned}
$$

From equations (14) and (15), we obtain:

$$
\left\{\begin{array}{l}
\dot{q}_{1}=\frac{\dot{C}_{X} \cos q_{2}+\dot{C}_{Y} \sin q_{2}}{l_{2} \sin \left(q_{1}-q_{2}\right)} \\
\dot{q_{4}}=\frac{\dot{D}_{X} \cos q_{3}+\dot{D}_{Y} \sin q_{3}}{l_{1} \sin \left(q_{3}-q_{4}\right)}
\end{array}\right.
$$

From equations (1), (2) and (10), (11), the calculated relationship between the end picking device shear hole centre point $T$ and the two points $C$ and $D$ can be obtained. Therefore, taking the derivative yields:

$$
\left\{\begin{array}{l}
\dot{q}_{1}=\frac{\dot{T}_{X} \cos q_{2}+\dot{T}_{Y} \sin q_{2}}{l_{2} \sin \left(q_{1}-q_{2}\right)} \\
\dot{q}_{4}=\frac{\dot{T}_{X} \cos q_{3}+\dot{T}_{Y} \sin q_{3}}{l_{1} \sin \left(q_{3}-q_{4}\right)}
\end{array}\right.
$$

A matrix transformation of [eq. (16)] yields:

$$
\left[\begin{array}{c}
\dot{q}_{1} \\
\dot{q_{4}}
\end{array}\right]=\left[\begin{array}{ll}
\frac{\cos q_{2}}{l_{2} \sin \left(q_{2}-q_{1}\right)} & \frac{\sin q_{2}}{l_{2} \sin \left(q_{2}-q_{1}\right)} \\
\frac{\cos q_{3}}{l_{1} \sin \left(q_{3}-q_{4}\right)} & \frac{\cos q_{3}}{l_{1} \sin \left(q_{3}-q_{4}\right)}
\end{array}\right]\left[\begin{array}{c}
\dot{T}_{X} \\
\dot{T}_{Y}
\end{array}\right]
$$

This result yields:

$$
\left[\begin{array}{c}
\dot{T}_{X} \\
\dot{T}_{Y}
\end{array}\right]=J_{T}\left[\begin{array}{c}
\dot{q}_{1} \\
\dot{q}_{4}
\end{array}\right]
$$

Comprehensive available $J_{T}$ :

$$
J_{T}=\left[\begin{array}{cc}
\frac{l_{2} \sin \left(q_{2}-q_{1}\right)}{\cos q_{2}-\sin q_{2}} & -\frac{l_{1} \sin \left(q_{3}-q_{4}\right) \sin q_{2}}{\cos q_{3}\left(\cos q_{2}-\sin q_{2}\right)} \\
-\frac{l_{2} \sin \left(q_{2}-q_{1}\right)}{\cos q_{2}-\sin q_{2}} & \frac{l_{1} \sin \left(q_{2}-q_{1}\right) \cos q_{2}}{\cos q_{3}\left(\cos q_{2}-\sin q_{2}\right)}
\end{array}\right]
$$

The speed analysis of a parallel mechanism is an important basis in mechanism performance analysis, dynamic analysis and motion control. Therefore, according to the calculation, the Jacobi matrix $J_{T}$ is obtained. The matrix shows that the speed of the end picking device and the parallel manipulator is only related to the input angle and rod length. Therefore, the speed of the parallel manipulator mechanism is controllable, and the analysis is simple and obtainable.

\section{Acceleration analysis}

Derivation of both sides of equation (17) with respect to time yields the acceleration at point $T$ at the centre of the end motion. The analysis yields: 


$$
\left\{\begin{array}{l}
\ddot{q_{1}}=\frac{\left(\dot{T}_{X} \cos q_{2}+\dot{T}_{Y} \sin q_{2}\right)^{\prime}\left[l_{2} \sin \left(q_{1}-q_{2}\right)\right]-\left(\dot{T}_{X} \cos q_{2}+\dot{T}_{Y} \sin q_{2}\right)\left[l_{2} \sin \left(q_{1}-q_{2}\right)\right]^{\prime}}{\left[l_{2} \sin \left(q_{1}-q_{2}\right)\right]^{2}} \\
\ddot{q_{1}}=\frac{\left(\dot{T}_{X} \cos q_{3}+\dot{T}_{Y} \sin q_{3}\right)^{\prime}\left[l_{1} \sin \left(q_{3}-q_{4}\right)\right]-\left(\dot{T}_{X} \cos q_{3}+\dot{T}_{Y} \sin q_{3}\right)\left[l_{1} \sin \left(q_{3}-q_{4}\right)\right]^{\prime}}{\left[l_{1} \sin \left(q_{3}-q_{4}\right)\right]^{2}}
\end{array}\right.
$$

simplified as:

$$
\left\{\begin{array}{l}
\ddot{q_{1}}=\frac{\left(\ddot{T}_{X}+l_{2} \cos q_{2} \dot{q}_{2}^{2}+l_{1} \cos q_{1} \dot{q}_{1}^{2}\right) \cos q_{1}-\left(\ddot{T}_{Y}+l_{2} \sin q_{2} \dot{q}_{2}^{2}+l_{1} \sin q_{1} \dot{q}_{1}^{2}\right) \sin q_{1}}{l_{2} \sin \left(q_{1}-q_{2}\right)} \\
\ddot{q_{4}}=\frac{\left(\ddot{T}_{X}+l_{1} \cos q_{4} \dot{q}_{4}^{2}+l_{2} \cos \dot{q}_{3} \dot{q}_{3}^{2}\right) \cos q_{3}-\left(\ddot{T}_{Y}+l_{1} \sin q_{4} \dot{q}_{4}^{2}+l_{2} \sin \dot{q}_{3} \dot{q}_{3}^{2}\right) \sin q_{4}}{l_{1} \sin \left(q_{3}-q_{4}\right)}
\end{array}\right.
$$

Where:

$$
\begin{aligned}
& \ddot{T_{X}}=-l_{1} \sin q_{1} \ddot{q_{1}}-l_{1} \cos q_{1} \dot{q}_{1}^{2}-l_{2} \sin q_{2} \ddot{q_{2}}-l_{2} \cos q_{2} \dot{q}_{2}^{2} \\
& \ddot{T_{Y}}=l_{1} \cos q_{1} \ddot{q_{1}}-l_{1} \sin {q_{1}}_{\dot{q}_{1}}{ }^{2}+l_{2} \cos q_{2} \ddot{q_{2}}-l_{2} \sin q_{2} \dot{q}_{2}^{2} .
\end{aligned}
$$

\section{Positive kinematics analysis}

The kinematic positive solution refers to the drive angles $q_{1}$ and $q_{4}$ of the active arm. According to the known centre dimensions of the fixed platform, centre dimensions of the moving platform and length of the branch chain rod, the forward solution is performed for the position coordinates $T(X, Y)$ of the centre point of the actuator at the end of the moving platform.

As shown in Figure 3, in ABCTDEF, the following set of equations can be derived from the kinematic relationship of the mechanism:

$$
\left\{\begin{array}{l}
l_{1} \cos q_{1}+l_{2} \cos q_{2}+2 l_{3}-l_{5} \cos q_{3}-l_{6} \cos q_{4}-l_{7}=0 \\
l_{1} \sin q_{1}+l_{2} \sin q_{2}-l_{5} \sin q_{3}-l_{6} \sin q_{4}=0
\end{array}\right.
$$

Assume that the coordinates of the centre point $\mathrm{T}$ of the moving platform are:

$$
\left\{\begin{array}{l}
T_{X}=l_{6} \cos q_{4}+l_{7}+l_{5} \cos q_{3} \\
T_{Y}=l_{6} \sin q_{4}+l_{5} \sin q_{3}+l_{4}
\end{array}\right.
$$

Where:

$\mathrm{q} 1$ and $\mathrm{q} 4$ are used as motion input parameters and $\mathrm{q} 2$ in equation (23) is eliminated to obtain the following equation:

$$
W+X+Y \cos q_{3}+Z \sin q_{3}=0
$$

Where:

$$
\begin{aligned}
& W=l_{1}^{2}+4 l_{3}^{2}+l_{5}^{2}+l_{6}^{2}+l_{7}^{2}-l_{2}^{2}-4 l_{3} l_{7} \\
& X=2\left[\left(l_{6} l_{7}-2 l_{3} l_{6}\right) \cos q_{4}+\left(l_{1} l_{7}-2 l_{1} l_{3}\right) \cos q_{1}+l_{1} l_{6} \cos q_{1} \cos q_{4}-l_{1} l_{6} \sin q_{1} \sin q_{4}\right] \\
& Y=2\left[\left(l_{5} l_{7}-2 l_{3} l_{5}\right)+l_{1} l_{5} \cos q_{1}+l_{5} l_{6} \cos q_{4}\right] \\
& Z=2\left(l_{5} l_{6} \sin q_{4}-l_{1} l_{5} \sin q_{1}\right)
\end{aligned}
$$


Let $\delta=\tan \frac{q_{3}}{2} ;$ then, $\cos q_{3}=\frac{1-\delta^{2}}{1+\delta^{2}}, \sin q_{3}=\frac{2 \delta}{1+\delta^{2}}$, and substituting $\delta$ into [eq. (25)] is known:

$(W+X+Y) \delta^{2}+2 Z \delta^{2}+(A+B-C)=0$

Solving [eq. (26)] yields $\delta=\frac{-Z \pm \sqrt{Z^{2}-(W+X+Y)(W+X-Y)}}{W+X+Y}$, and after finding $\delta$, according to $\delta=\tan \frac{q_{3}}{2}$, we can obtain:

$$
q_{3}=2 \arctan \delta
$$

The active input parameters $\mathrm{q} 1$ and $\mathrm{q} 4$ and [eq. (27)] are applied to obtain the attitude angle $\mathrm{q} 3$, and the position coordinates of the centre $\mathrm{T}$ of the moving platform can be obtained by substituting them into [eq. (24)]. Therefore, the posture-positive solution equation set of the reference coordinate $\mathrm{T}$ can be obtained by the [eq. (28)] representation.

$$
\left\{\begin{array}{l}
T_{X}=l_{6} \cos q_{1}+l_{7}+l_{5} \cos q_{3} \\
T_{Y}=l_{6} \sin q_{4}+l_{5} \sin q_{3}+l_{4} \\
q_{3}=2 \arctan \delta
\end{array}\right.
$$

\section{Singularity analysis}

Singularity is an unavoidable phenomenon that occurs in all mechanisms. The concept of a singular position of the mechanism can be summarized as follows: the mechanism moves under the drive of the active part, and if the kinematic and dynamic properties of the mechanism change suddenly and instantaneously during the movement, the mechanism is either at a dead point or loses stability, or the degrees of freedom change, which makes the ability of the mechanism to transmit motion and power out of order. At this time, the position shape of the mechanism is referred to as a singular position. For the parallel mechanism, there are two types of singularities.
First, when the mechanism is in a specific locus, its static Jacobi matrix becomes a singular array, and its determinant value is zero. When the inverse solution of the mechanism does not exist, this locus is an unstable singular locus, and the robot is in an uncontrollable state at this time. Second, the static Jacobi matrix does not descend in rank, but the degrees of freedom of the motion platform change. Although the mechanism is stable, the number or nature of its degrees of freedom changes, causing continuous motion in some directions that cannot be achieved.

One derivative of the system of eqs (23) and (24), respectively, yields the Jacobi matrix $\mathrm{J}$ for the position equation:

$$
J=\left[\begin{array}{cccc}
-l_{1} \sin q_{1} & -l_{2} \sin q_{2} & l_{5} \sin q_{3} & l_{6} \sin q_{4} \\
l_{1} \sin q_{1} & l_{2} \cos q_{2} & -l_{5} \cos q_{3} & -l_{6} \cos q_{4} \\
0 & 0 & -l_{5} \sin q_{3} & -l_{6} \sin q_{4} \\
0 & 0 & l_{5} \cos q_{3} & l_{6} \cos q_{4}
\end{array}\right]
$$
follows:

Let the value of the determinant of $\mathrm{J}$ be 0 . After simplification, the workspace boundary equation can be obtained as

$$
l_{1} l_{2} \sin \left(q_{2}-q_{1}\right)=l_{5} l_{6} \sin \left(q_{4}-q_{3}\right)=0
$$

To satisfy [eq. (30)], it is necessary that 11 and 12, 15 and 16 are straightened or overlapped, namely:

$$
q_{2}-q_{1}=\pi \text { or } q_{2}-q_{1}=0 ; q_{4}-q_{3}=\pi \text { or } q_{4}-q_{3}=0
$$

Figure 2 and Figure 3 show that the two active arms of the parallel robot arm are symmetrical, so the working boundary (swing range) of the two active arms is also symmetrical, and only the working boundary of one active arm. Consider the active arm $\mathrm{AB}$ as an example: when the active arm $\mathrm{AB}$ swings anticlockwise to the angle of zero with the driven arm $\mathrm{BC}$, the main and driven arms interfere, and the position of the active arm $\mathrm{AB}$ at this time is its upper limit position. Its lower limit position can only be 11 and 12 forward common lines (straightened), that is, $\mathrm{q} 2-\mathrm{q} 1=\pi ; \mathrm{ABCDE}$ is transformed from a five-bar mechanism into a four-bar mechanism; the corresponding common line position appears dead, that is, the transmission angle is zero; and the position of the active arm is its working boundary. 


\section{Error modelling and accuracy analysis}

The manufacturing and assembly process of the mechanical structure will produce certain geometric errors, a certain deviation between the kinematic model entered in the upper computer and the kinematic model in reality, and a deviation between the position obtained by using the drive joint motor of the ideal kinematic model of the parallel robot arm and the actual position of the endmotion platform (Dai et al., 2016). Studies have shown that the vast majority of the robot's absolute positioning errors are caused by an inaccurate description of the kinematics model inside the robot (Judd \& Knasinski, 1990). Therefore, it is necessary to determine the motion accuracy of the moving platform by error modelling and accuracy analysis of the parallel manipulator.

\section{Error modelling}

The positive kinematics analysis is established by the 2-DOF parallel manipulator, and the error model is established on this basis. The driving angle is considered the main research object of the study to observe the influence of the deviation of the end moving platform position when it generates errors.

In the established plane coordinate system $O-X Y, T(X$, $Y$ ) is the centre position of the end moving platform and is taken as the target point for error analysis, which is shown in Figure 4.

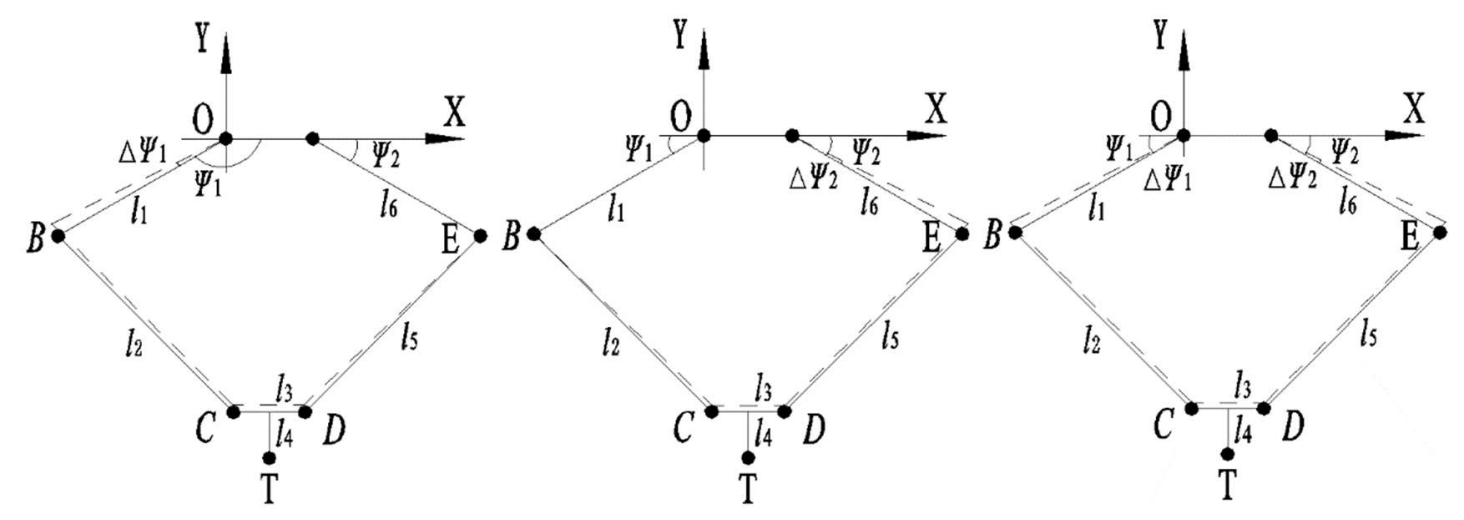

FIGURE 4. Error analysis diagram in three cases.

$\psi_{i}{ }^{\prime}=\psi_{i}+\Delta \psi(i=1,2)$ refers to the driving angle of the active arm in the presence of an error value, and the error parameter is input into the positive solution equation of each branch chain to obtain the following equations:

$$
\begin{aligned}
& \left\{\begin{array}{l}
F_{1}=\left(l_{1} \cos q_{1}+T_{X}-l_{3}\right)^{2}+ \\
\left(T_{Y}+l_{4}-l_{1} \sin q_{1}\right)^{2}-l_{2}^{2}=0 \\
F_{2}=\left(l_{6} \cos q_{4}+l_{7}-T_{X}-l_{3}\right)^{2}+ \\
\left(T_{Y}+l_{4}-l_{6} \sin q_{4}\right)^{2}-l_{5}{ }^{2}=0
\end{array}\right. \\
& \left\{\begin{array}{l}
F_{1}{ }^{\prime}=0 \\
F_{2}{ }^{\prime}=0
\end{array} \Rightarrow F_{i}{ }^{\prime}=\left[F_{1}{ }^{\prime}, F_{2}{ }^{\prime}\right]^{T}=[0,0]^{T}\right.
\end{aligned}
$$

The nonlinear equations of the error model are jointly solved to obtain the position coordinate $T^{\prime}\left(T X^{\prime}, T Y^{\prime}\right)$ of the centre point of the end moving platform, and then the centre position coordinate $T(X, Y)$ under ideal conditions is solved. A further calculation is presented as follows:

$$
\Delta T=\sqrt{\left(T_{X}{ }^{\prime}-T_{X}\right)^{2}+\left(T_{Y}{ }^{\prime}-T_{Y}\right)^{2}}
$$

\section{Error analysis of the driving angle}

The parallel robot in this design has two coupled branch chains, where each branch chain is driven by a separate motor, so the drive angle of the active arm of each branch chain is the main factor that generates errors. Considering the drive angle error value $\Delta \psi$ in the range of $0.001^{\circ} \sim 0.005^{\circ}$ and substituting it into the error model, $\Delta T_{i}(i=1,2)$ represents the end position error when the drive angles of the two branch chains separately generate errors. $\Delta T$ represents the position error when the two branch chains simultaneously generate errors, and after calculating the position error, it further analyses its impact on the end position control accuracy when it generates errors. The calculation results are shown in Table 1. 
TABLE 1. Data of the drive angle error.

\begin{tabular}{ccccccc}
\hline Error value of drive angle $\Delta \psi\left({ }^{\circ}\right)$ & 0.001 & 0.002 & 0.003 & 0.004 & 0.005 \\
\hline$\Delta T_{1}$ & 0.1750 & 0.3500 & 0.5250 & 0.700 & 0.8750 \\
$\Delta T_{2}$ & 0.1502 & 0.2702 & 0.3856 & 0.5051 & 0.6232 \\
$\Delta T$ & 0.2174 & 0.3928 & 0.5718 & 0.7598 & 0.9387 \\
\hline
\end{tabular}

\section{Curve of the drive angle error}

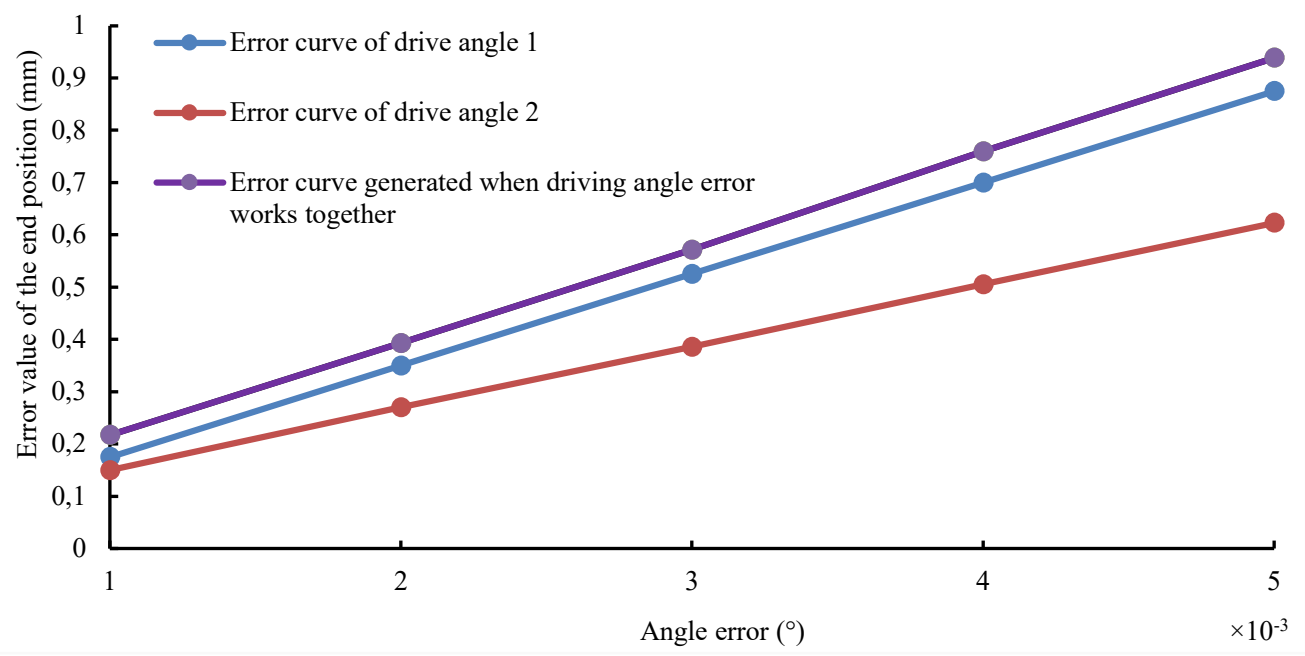

FIGURE 5. Curve of drive angle error.

The analysis of the data in Table 1 indicates that when the two driving angles simultaneously produce errors, the resulting end position error is the largest, and when the error value is taken as $0.001^{\circ}$, the minimum error of the end position is $0.2174 \mathrm{~mm}$. When the error value is $0.005^{\circ}$, the maximum error of the end position is 0.9387 $\mathrm{mm}$. According to Figure 4, the end position error tends to gradually increase as the drive angle error value increases. The analysis of the results reveals that within the range of the drive angle error value from $0.001 \sim 0.005^{\circ}$, the calculated ideal motion accuracy of the parallel mechanism ranges from $0.2174-0.9387 \mathrm{~mm}$, which has a small impact on the work effect of safflower picking and meets the position accuracy requirements of the test phase.

\section{Workspace Analysis}

Workspace analysis is an important part of the design of parallel mechanisms, where the workspace refers to the working area of the end effector of the robot, and there are usually reachable working spaces and flexible working spaces (Feng et al., 2014). The workspace analysis of the parallel manipulator is performed to derive the workspace of the end picking device of the picking robot, which is employed to judge the rationality of the mechanical design of the parallel manipulator. Therefore, the workspace analysis of the parallel robotic arm is an important indicator for evaluating whether the saffron picking robot can accomplish the picking task. The solution of the workspace of the parallel mechanism depends on its motion equations, and the available numerical methods include the grid method, Jacobi method, and Monte Carlo method (Cai, 2009; Cong \& Shang 2010). Based on MATLAB simulation software, this paper used a parallel manipulator operability analysis method that is based on the Monte Carlo method (Zhang et al., 2013) and obtained the workspace of the parallel robotic arm by calculation and analysis, as shown in Fig. 6. 


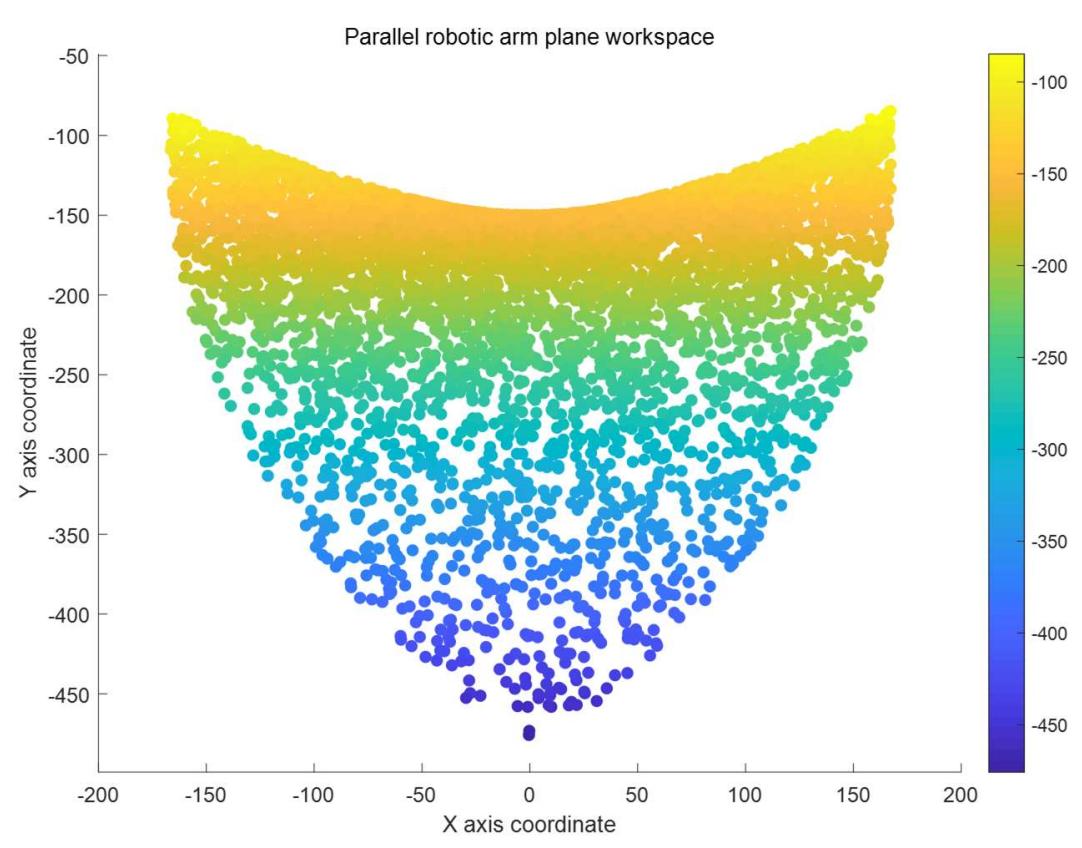

FIGURE 6. Parallel manipulator workspace diagram.

According to the simulation results, the planar working space of the parallel manipulator is approximately an equilateral triangle with a side length of $0.35 \mathrm{~m}$. With regard to the growth characteristics of the safflower, plant height and horizontal plant coverage area, the working space of the robot arm designed in this paper basically meets the requirements and verifies the reasonableness of the mechanical design of the parallel manipulator.

\section{Picking Test}

\section{Test design}

Based on the mechanism analysis and simulation results, the preliminary feasibility of the experiment was obtained. Thus, a prototype of a safflower picking robot was built based on a parallel manipulator. The test was carried out in the laboratory of the School of Mechanical and Electrical Engineering of Xinjiang Agricultural University. Due to the particularity of the test time, the test safflower was planted under laboratory conditions, and Xinjiang thornless safflower was selected for planting. In each special flowerpot, approximately 1-4 safflowers were planted, and a total of 15 pots of safflower were selected for the experiment. The height of the safflower plant ranges from $0.43-0.86 \mathrm{~m}$ (for the ground), the crown width ranges from $0.14-0.38 \mathrm{~m}$, and the picking test is shown in Fig. 7.

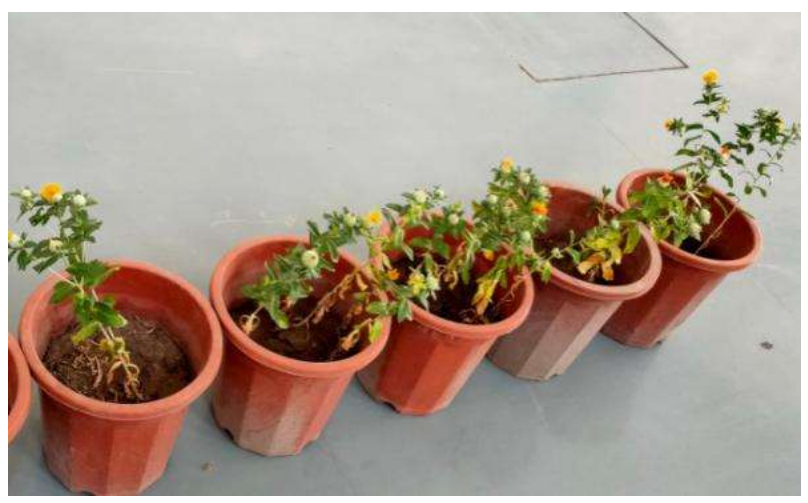

(a) Before picking

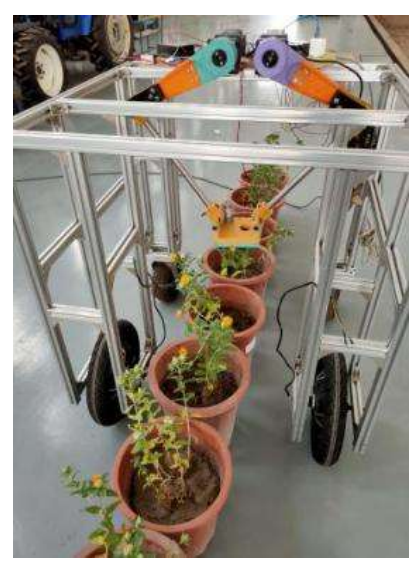

(b) Picking

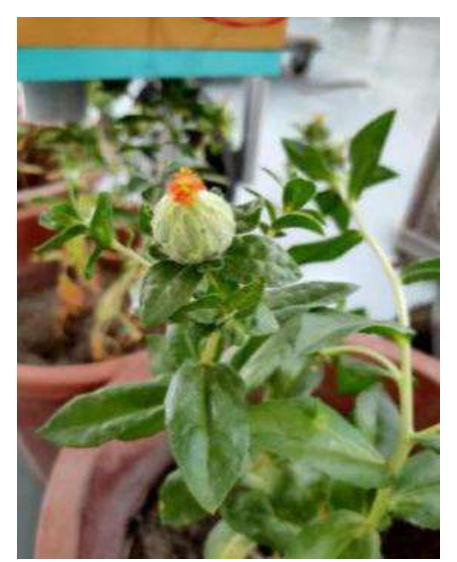

(c)After picking

FIGURE 7. Picking test

\section{Test conditions}

The test equipment included a safflower picking robot (homemade) that is based on a parallel manipulator, in which the picking device included a 25GA-370 DC geared motor (reduction ratio of 21.3 and rotation speed of 180 $\mathrm{rpm}$ ), a crank slider shearing device (reciprocating 
shearing speed $30 \mathrm{~mm}) / \mathrm{s})$, LX-8001 negative pressure suction fan (wind speed $85 \mathrm{~m} / \mathrm{s}$ and rotation speed 30000 $\mathrm{r} / \mathrm{min}$ ), JM-B1003 electronic balance (range of $100 \mathrm{~g}$ and accuracy of $0.001 \mathrm{~g}$ ), DT-2235B + tachometer (range from 0.5-19999 rpm; resolution of $0.1 \mathrm{rpm}$ ), sealed bag, tweezers, etc.

\section{Analysis of picking cycle and net filament picking rate}

The picking cycle refers to the time from the end of the picking of the last flower ball to the end of the picking of the current flower ball, which is recorded by a stopwatch. The calculation expression is:

$$
T=\frac{\sum_{i=1}^{n} T_{n}}{n}
$$

in which:

$T$ - average picking cycle, $s$;

$T_{n}$ - picking time of each flower ball, $s$;

$n$ - total number of flower balls.

The filament picking rate refers to the weight of the filaments cut and collected in the filament collection box by the picking device and the ratio of the total weight of the filaments in the entire experiment, where the total weight refers to the sum of the weight of the filaments that have not been cut to be left on the flower ball, dropped filaments and the collection box. The calculation expression is as follows:

$$
P=\frac{m_{1}}{m_{1}+m_{2}+m_{3}} \times 100 \%
$$

in which:

$$
\begin{aligned}
& P \text { - net collection rate of filaments, } \% \\
& m_{1}-\text { weight of filaments in the filament collection } \\
& \text { box, } \mathrm{g} \text {; } \\
& m_{2} \text {-weight of filaments on the flower ball, } \mathrm{g}, \\
& m_{3} \text { - weight of dropped filaments, } \mathrm{g} \text {. }
\end{aligned}
$$

The 15 pots of plants were equally divided into groups of 3 pots for a total of 5 groups of test samples. The picking test was carried out under laboratory conditions, and the test results are shown in Table 2.

TABLE 2. Results of safflower picking tests.

\begin{tabular}{cccc}
\hline Number & Weight of filaments in the collection box & Total weight of the filaments & Net collection rate \\
\hline 1 & 2.583 & 2.973 & $86.88 \%$ \\
2 & 3.087 & 3.607 & $85.58 \%$ \\
3 & 2.62 & 2.94 & $89.12 \%$ \\
4 & 1.983 & 2.233 & $88.80 \%$ \\
5 & 2.139 & 2.399 & $89.16 \%$ \\
\hline
\end{tabular}

\section{RESULTS AND DISCUSSION}

The test results showed that the average picking cycle of safflower filaments was $16 \mathrm{~s} /$ flower ball, and the average net picking rate of filaments was $87.91 \%$, which satisfied the filament picking requirements and verified the feasibility of the safflower picking robot. During the test, uncollected and dropped filaments were noted. Compared with manual picking, the net picking rate of the prototype needs to be improved. As the device is still in the testing stage, real mechanized, automated, and intelligent picking is not yet fully realized, the picking cycle time should be shortened, and the net recovery rate needs to be improved. Therefore, it is necessary to further optimize the parameters of each device of the prototype, which provides a reference for later test improvement.

\section{CONCLUSIONS}

A safflower picking robot based on a parallel manipulator is proposed, which has a simple and compact structure and a reasonable layout. The robot can realize a series of actions, such as safflower recognition, positioning, coordinate conversion, and picking and collection.
By establishing kinematic equations for the parallel robotic arm mechanism, the coordinate transformation conditions of the robot are obtained; the velocity analysis of the picking device based on the Jacobi matrix is conducted; the error model of the robotic arm is established, and an accuracy analysis is carried out. Additionally, a motion accuracy of $0.2174-0.9387 \mathrm{~mm}$ is determined within a driving angle error of $0.001^{\circ} \sim 0.005^{\circ}$, which meets the position accuracy requirements of the test phase. Based on the MATLAB simulation software, the Monte Carlo method is utilized to obtain the working space of the parallel manipulator, which is approximated as an equilateral triangle with a side length of $0.35 \mathrm{~m}$. The rationality of the workspace is determined by comparing the growth space of safflower.

A safflower picking robot prototype based on a parallel manipulator is built. To verify the picking performance of this safflower picking device, experiments are carried out in a laboratory environment. The test results showed that the average period of safflower picking was $16 \mathrm{~s}$ and that the average net picking rate of filaments was $87.91 \%$. 


\section{ACKNOWLEDGEMENTS}

This study was supported by China's natural science foundation No. 31901417. Funding was provided by the Ministry of Science and Technology of China. The author also thanks Associate Professor Zhang Zhengguo and General Master Guo Yanke for their assistance in this study.

\section{REFERENCES}

An LL, Cao WB, Lian GD, Ma P, Yang SP, Niu C (2020) Design and test of comb-type safflower picking test bench. Journal of Agricultural Mechanization Research42 (10): 109-112, 199.

Anil KR (2005) Development of Safflower Petal Collector. Nimbkar Agricultural Research Institute (NARI):1-6.

Bai YW, Li X, Shao YQ, Lv QF, Yang QH (2020) Design and research of cropping and grasping safflower picking device. Mechanical Engineer (11): 54-58.

Cai ZX (2009) Fundamentals of robotics. Beijing, China Machine Press.

Cao WB, Sun WL, Niu C, Jiao HB, Chen BB (2018) Research on comb clip safflower picking device based on ANSYS / LS-DYNA. Transactions of the Chinese Society for Agricultural Machinery49 (11): 123-131.

Cong S, Shang WW (2010) Parallel robots: Modeling, control optimization and applications. Beijing, Publishing House of Electronics Industry.

Dai ZW, Liu C, Sheng XJ, Zhang DG (2016) The Error Analysis and Calibration of Delta Parallel Robot. Electromechanical integration 22(3): 8-12.

Feng LH, Zhang WG, Gong ZY, Lin GY, Liang DK (2014) Research progress and status quo of delta series parallel robot. Robot36 (03): 375-384

Han DD (2014) Design and airflow field simulation of pneumatic safflower silk harvesting device. Thesis, Shi He Zi University.

Hoevenaars AGL, Krut S, Herder JL (2020) Jacobianbased natural frequency analysis of parallel manipulators. Mechanism and Machine Theory 148: e:103775. DOI: https://doi.org/10.1016/j.mechmachtheory.2019.103775

Huang Z, Kong LF, Fang YF (1997) Mechanism theory and control of parallel robot. Beijing, China Machine Press.

Judd RP, Knasinski AB (1990) A technique to calibrate industrial robots with experimental verification. Robotics and Automation, IEEE Transactions on 6(1): 20-30

Kuo YL, Tang SC, Gosselin C, Angeles J, Lee KM, Yien C (2021) Dynamics and control of a 3-DOF planar parallel manipulator using visual servoing resolved acceleration control. Journal of Low Frequency Noise. Vibration and Active Control 40 (1): 458-480

Li HJ, Li L (2011) Timely harvesting and drying method of safflower. Rural Bestone (12): 19-20.
Lian GD, Cao WB, Niu C, An LL, Yang SP, Li LL (2019) Design and analysis of key components of comb-type safflower picking mechanism. Machine Design \& Research 35(2): 187-190.

Liu GX, Ge Y, Zhang LX, Zhao YZ, Zhang TY (2019) Cutting-type safflower picking test bench design and picking performance test. Machine Design \& Research 35(2): 178-183.

Ma XL, Ma LZ, Zhou ZZ, Zhou JQ (2006) Structural synthesis of parallel mechanism based on branch chain degree of freedom. Transactions of the Chinese Society for Agricultural Machinery (2): 97-101.

Mc Guire PE, Damania AB, Qualset CO (2012) Agronomy Progress Report No. 313, ept. of Plant Sciences. University of California.

Siavash A, Gholamreza C, Mohamad H K, Adel H (2012) Design and construction of a harvesting safflower petals machine. Mechanics \& Industry 13(5): 301-305.

State Pharmacopoeia Committee (2010) Chinese pharmacopoeia. Beijing, China, Medical Science and Technology Press, p 141-142.

Sun WL, Cao WB, Gu LL, Liu JD, Wang SH (2018) Design and validation of gripping picking mechanism based on safflower mechanical properties. Journal of Agricultural Mechanization Research 40 (5):46-51.

Wang KQ, Zhang WH, Luo Z, Zhang YZ (2020) Design and experiment of percussion pine picking robot. Transactions of the Chinese Society for Agricultural Machinery 51(8): 26-33.

Zhang J, Xu T, Fang HL, Zhang LA (2020) Kinematics analysis of a kind of delta robot with 6 degrees of freedom. Transactions of the Chinese Society for Agricultural Machinery 51(3): 419-426.

Zhang JF, Wang JJ, Feng PF, Li TM (2013) Monte Carlo method for operability analysis of parallel robots. Transactions of the Chinese Society for Agricultural Machinery 44(7): 269-273.

Zhang TY, Zhang LX, Ge Y, Liu GX, Zhang XW (2020) Development and test of a portable roll-type safflower harvester. Journal of Agricultural Mechanization Research 42(3): 76-81.

Zhang ZG, Lv QG, Ren JY, Han CJ, Yuan PP, Zhang XJ (2019) Design of key components of rotary shear safflower silk picking machine. Journal of Chinese Agricultural mechanization 40(7): 1-6.

Zhao QQ, Guo JK, Zhao DT, Yu DW, Hong J (2020) A Novel Approach to Kinematic Reliability Analysis for Planar Parallel Manipulators. Journal of Mechanical Design 142(8). 